www.nature.com/pj

\title{
Application of the simultaneous measurement system of WAXD, SAXS and transmission FTIR spectra to the study of structural changes in the cold- and melt- crystallization processes of trans-1,4-polyisoprene
}

\author{
Paramita Jaya Ratri and Kohji Tashiro
}

The newly developed system of simultaneous time-dependent measurements of wide-angle X-ray diffraction, small-angle X-ray scattering and transmission-type Fourier-transform infrared spectra has been applied to study the structural changes in the melt- and cold-crystallization processes of trans-1,4-polyisoprene (TPI). For the melt-isothermal crystallization process at $40^{\circ} \mathrm{C}$, the domains of relatively higher density with approximately $220 \AA$ radius formed at first as the intermediate state, where the chain conformation was speculated to be considerably disordered. As time passed, the correlation length $\xi$ between these domains decreased until they joined together to form the stacked lamellar structure. In these lamellae, the crystal lattices of the regular $\alpha$ form were created. When the isothermal crystallization was investigated at $30^{\circ} \mathrm{C}$, the intermediate-phase regularization caused the $\alpha$ - and $\beta$-crystalline forms to mix. In the cold-crystallization process or the heating process that started from the melt-quenched glass below $-100^{\circ} \mathrm{C}$, TPI was found to crystallize at first to the $\beta$-form at approximately $-55{ }^{\circ} \mathrm{C}$, which transformed to the $\alpha$-form at $50^{\circ} \mathrm{C}$ via the amorphous phase as observed above.

Polymer Journal (2013) 45, 1019-1026; doi:10.1038/pj.2013.31; published online 24 April 2013

Keywords: crystallization; structure evolution; trans-1,4-polyisoprene

\section{INTRODUCTION}

Recently, we developed a system of the simultaneous and timeresolved measurement of wide-angle X-ray diffraction (WAXD), small-angle X-ray scattering (SAXS) and transmission-type FTIR spectra during the application of external fields such as temperature and tensile stress. ${ }^{1-3}$ This system can provide information about the structural evolution process of synthetic polymers viewed from various hierarchical levels. The infrared spectral data show the regularization of the molecular chain conformation. This spectroscopic data can be combined with the WAXD data to reveal the crystalline lattice formation with the ordered or disordered packing of these chains. The SAXS data, which show the creation process of stacked lamellae, must also be discussed in relation to this molecular-level information. In previous works, we reported several case studies including the tensile-force-induced phase transition of poly(tetramethylene terephthalate), ${ }^{1}$ high-temperature phase transition of aliphatic nylons ${ }^{2}$ and isothermal crystallizations of poly(L-lactic acid) ${ }^{1}$ and polyethylene. ${ }^{3}$

In this paper, we will treat the structural evolution processes in the crystallization phenomena of trans-1,4-polyisoprene (TPI), which is well known as Gutta Percha and is one of the typically used biodegradable green polymers in various fields to produce artificial bone, the tooth root canal cone, golf balls and so on ${ }^{4-16}$ (http:// www.hitzbio.com/research\%20E.html). However, the structural information, which is fundamentally important to study the microscopically viewed crystallization phenomenon, has not been well established. ${ }^{17-34}$ To further develop this polymer in the recent field of green polymer science, we need to clarify the structural information in detail.

TPI exhibits several crystalline modifications ( $\alpha, \beta$ and so on) depending on the preparation conditions. Recently, we refined the crystal structures of the typical $\alpha$ - and $\beta$-forms based on X-ray diffraction data analyses. ${ }^{17-20,35}$ We discovered new methods to prepare these crystal forms with the highest possible purity and orientation, which gave many X-ray diffraction spots to produce accurate crystal structures. ${ }^{35}$ In the study of the preparation methods of pure crystalline forms, we noticed that TPI exhibited various types of transition behaviors in the heating and cooling procedures, in particular the crystallization behaviors, which have not been clarified in enough details for this polymer.

Regarding the crystallization behavior of TPI, only the macroscopic viewpoint has been reported. ${ }^{21,25-29}$ For example, Cooper and Vaughan ${ }^{29}$ measured the dilatometry in the isothermal crystallization and estimated the Avrami index, and Fischer and Henderson ${ }^{21}$ 
studied the formation of spherulites from the melt using a polarized optical microscope. However, the study of crystallization viewed from the molecular level has not been reported. In the present paper, the structural evolution of TPI was studied in the crystallization processes from the melt and glass based on the above-mentioned simultaneous measurement system of WAXD, SAXS, FTIR spectra and the thermal analysis using a differential scanning calorimeter (DSC). The crystallization experiments were performed in different manners: (i) the non-isothermal cooling process from the melt as a preliminary study, during which the FTIR spectra were measured; (ii) the isothermal crystallization process from the melt to the predetermined crystallization temperature, during which WAXD, SAXS and FTIR spectra were simultaneously measured; and (iii) the coldcrystallization process from the glass, which was obtained by quenching the melt into liquid nitrogen temperature, and during this process, the WAXD and SAXD measurements were performed simultaneously as a function of temperature.

\section{EXPERIMENTAL PROCEDURE}

\section{Samples}

A synthetic TPI sample with $99 \%$ trans content was purchased from Polyscience Inc. (Warrington, PA, USA). The films of approximately $100 \mu \mathrm{m}$ thickness were prepared by pressing the melt at $115^{\circ} \mathrm{C}$. The number-averaged and weight-averaged molecular weights of the sample were 53000 and 400000 , respectively.

\section{Measurements}

Non-isothermal crystallization. To examine the crystallization behavior of TPI in a preliminary experiment, the temperature-dependent measurement was initially performed with an FTIR spectrometer. The spectrometer that we used was a Varian FTS7000 FTIR spectrometer (Varian, Santa Clara, CA, USA) at a resolution power of $2 \mathrm{~cm}^{-1}$. The sample was set in a homemade heater with a pair of $\mathrm{KBr}$ plates as the window material.

Melt-isothermal crystallization. The structural changes in the isothermal crystallization process from the melt were investigated using the simultaneous measurement system of WAXD, SAXS and FTIR spectra. ${ }^{1}$ The temperature jump cell was mounted on a sample stage, around which a portable FTIR spectrometer (Alpha; Bruker, Germany) was set to measure the transmissiontype IR spectra. The WAXD data were measured using a two-dimensional (2D) photon counter Pilatus 100k (Dectris, Switzerland), which was set approximately $132 \mathrm{~mm}$ from the temperature jump cell window. The SAXS data were detected using a 2D photon counter Pilatus 300k (Dectris) at a distance of $1048 \mathrm{~mm}$ from the sample position. The X-ray generator that we used was a Rigaku Nanoviewer (Rigaku, Tokyo, Japan) $(40 \mathrm{kV}, 30 \mathrm{~mA})$ with a Cu-rotating target and a pair of confocal mirrors to produce a focused beam of approximately $200 \mu \mathrm{m}$ in diameter, where the X-ray wavelength was $1.5418 \AA$. In the temperature jump process, the WAXD and SAXS data were collected every $1 \mathrm{~min}$. The IR spectra were measured every $30 \mathrm{~s}$ at a resolution power of $2 \mathrm{~cm}^{-1}$.

The details of the temperature jump cell were described in the literature. ${ }^{3}$ The cell consists of three boxes, a high-temperature box (TA), a cooling box (TB) and a low-temperature box (TC). The sample was set in a small metal holder with an embedded thermocouple to monitor the actual temperature within the sample. The sample in the TA box was heated to above the melting point and melted for a predetermined time to erase all of the sample preparation history. After the prefixed time, the jump cell was moved and the sample holder touched the cooling box $\left(\mathrm{TB}\right.$ at $\left.0^{\circ} \mathrm{C}\right)$ when the temperature of the sample quickly decreased at a rate of approximately $600{ }^{\circ} \mathrm{C} \mathrm{min}-1$. When the temperature of the sample almost reached the crystallization temperature $T_{\mathcal{C}}$, the cell was moved again so that the low-temperature box (TC) touched the sample holder. These movements of the cell were controlled using a computer program that was written with a Lab viewer. The X-ray beam constantly passed through the sample holder, and the WAXD and SAXS patterns were measured repeatedly during the crystallization at $T_{c}$. The FTIR spectra were measured at the same time.
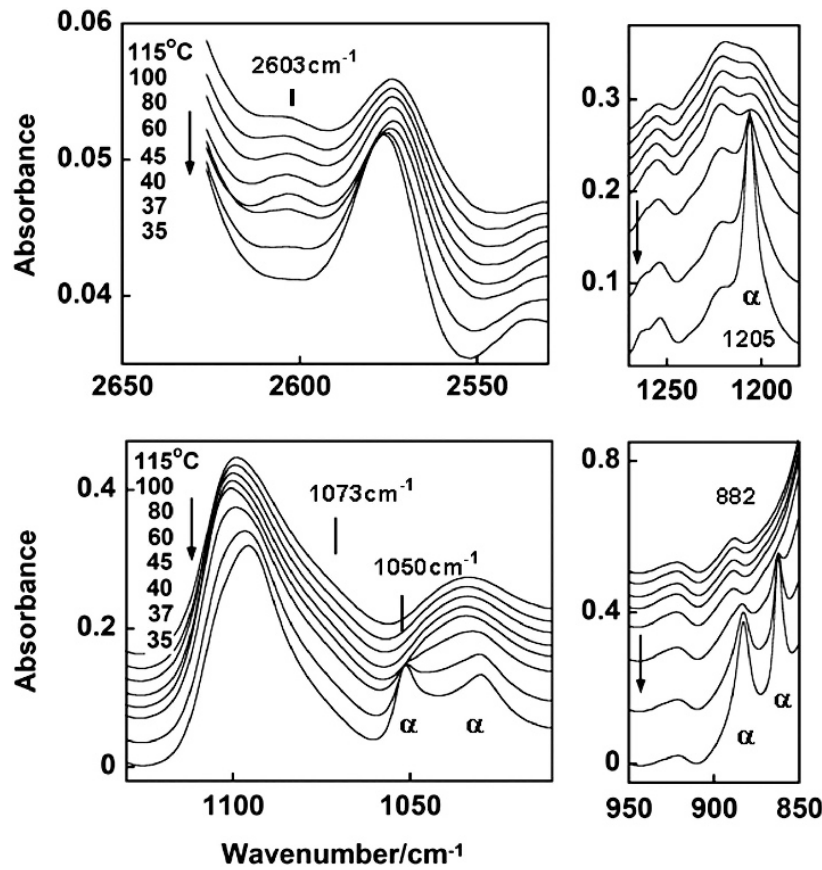

Figure 1 Temperature dependence of the infrared spectra of TPI measured in the cooling process from the melt.

Thermal analysis of isothermal crystallization. The enthalpy change during the isothermal crystallization process was measured using a DSC instrument (TA, DSC Q1000). The sample was placed in an aluminum pan and heated to a temperature above the melting point on a hot plate near the DSC thermometer. After a predetermined time, the pan was quickly placed on the sample stage of the DSC cell, where the temperature of the sample stage was constantly maintained at the crystallization temperature $T c$. In this process, the enthalpy change was measured as a function of time, from which the crystallization rate was estimated.

Cold crystallization. The TPI sample was quenched at liquid nitrogen temperature, and the amorphous glass sample was obtained. The 2D WAXD and SAXS patterns were simultaneously measured in the heating process using the above-mentioned system.

\section{RESULTS AND DISCUSSION}

\section{Non-isothermal crystallization}

The structural change in the cooling process from the melt was investigated using the temperature-dependent measurement of the FTIR spectra. As shown in Figure 1, during the stepwise cooling process from the molten state $\left(115^{\circ} \mathrm{C}\right)$ to room temperature, the bands at $1205,1050,882 \mathrm{~cm}^{-1}$ and so on, increased in intensity, whereas the bands at 1225 and $1073 \mathrm{~cm}^{-1}$ decreased in intensity. They were classified as the crystalline and amorphous bands, respectively. The bands in the frequency region of $2650-2530 \mathrm{~cm}^{-1}$ exhibited different behaviors from the above-mentioned bands. For example, the band at $2603 \mathrm{~cm}^{-1}$ was weakly detected above the melting point. As the temperature decreased, the intensity gradually increased. When the temperature decreased to approximately $40^{\circ} \mathrm{C}$, the band intensity decreased again and finally disappeared. At this moment, the bands of the crystalline $\alpha$-form started to appear and increased in intensity. Figure 2 shows the temperature dependence of the integrated intensities estimated for the bands at 2603, 1225 and $1205 \mathrm{~cm}^{-1}$. From these observations, we may conclude that the melt changed once to an intermediate phase and then to the $\alpha$-crystalline 


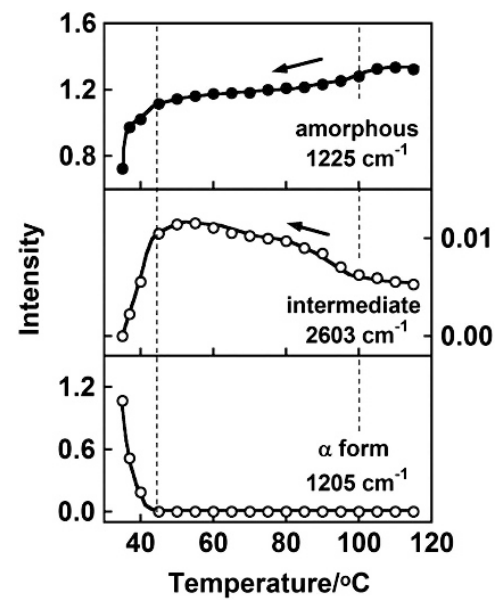

Figure 2 Temperature dependence of the integrated intensity of the TPI infrared bands at 2603 (intermediate phase), 1225 (amorphous phase) and $1205 \mathrm{~cm}^{-1}(\alpha$-form) in the non-isothermal crystallization process (refer to Figure 1).

form in the cooling process. However, the details of the intermediate phase were not clear.

\section{Isothermal crystallization phenomena}

Isothermal crystallization studied by DSC. The enthalpy change in the isothermal crystallization process was measured at various temperatures $\left(T_{c}\right)$, and the normalized degree of crystallinity $X(t)$ was estimated as a function of time $t$ using this enthalpy change. The Avrami plots were produced based on the following equation. ${ }^{36-41}$

$$
\log [-\ln (1-X(t))]=n \log (k)+n \log (t)
$$

From these plots, the Avrami constants $k$ and $n$, or the crystallization rate constants $(k)$ and the crystallization dimensions $(n)$, were evaluated. The $n$ value was 2.41 (at $25^{\circ} \mathrm{C}$ ), 2.50 (at $30^{\circ} \mathrm{C}$ ) and 2.45 (at $40^{\circ} \mathrm{C}$ ), which agreed with the literature values ${ }^{29}$ and indicated that the crystallization occurred in the 1-2D mode if we assumed a homogeneous crystallization, and in the $2-3 \mathrm{D}$ mode if we assumed a heterogeneous crystallization. The $k$ values were $1.17 \times 10^{-2} \mathrm{~s}^{-1}$ (at $25^{\circ} \mathrm{C}$ ), $0.32 \times 10^{-2} \mathrm{~s}^{-1}$ (at $30^{\circ} \mathrm{C}$ ) and $0.03 \times 10^{-2} \mathrm{~s}^{-1}$ (at $40^{\circ} \mathrm{C}$ ).

Simultaneous measurement of SAXS/WAXD/FTIR spectra. The isothermal crystallization experiment was performed at $T_{\mathrm{c}}$ of 30 and $40^{\circ} \mathrm{C}$, during which the SAXS, WAXD and FTIR spectra were measured simultaneously as a function of time as shown in Figures 3-5 with $T c=40^{\circ} \mathrm{C}$, respectively. The $1 \mathrm{D}$ X-ray diffraction profiles were derived from the $2 \mathrm{D} \mathrm{X}$-ray data using circular integration as a function of $2 \theta$.

(A) $T_{c}=40^{\circ} \mathrm{C}$

The starting time $t=0 \mathrm{~min}$ was defined as the time point where the temperature reached $T_{\mathrm{c}}$. As shown in Figure 4 , the WAXD pattern at the starting point was of the amorphous halo. The crystalline peaks of the $\alpha$-form started to increase the intensity at approximately $30 \mathrm{~min}$ after the temperature jump. The integrated intensities of 110 reflections and the amorphous halo are plotted against time in a logarithmic scale in Figure 6. As observed in Figure 5, the infrared band at $2603 \mathrm{~cm}^{-1}$ appeared first, and then the bands of the $\alpha$-form at 1050 and $882 \mathrm{~cm}^{-1}$ appeared and increased the intensity at approximately the same time as the WAXD peaks, as observed in Figure 6. These observations showed that the intermediate phase,
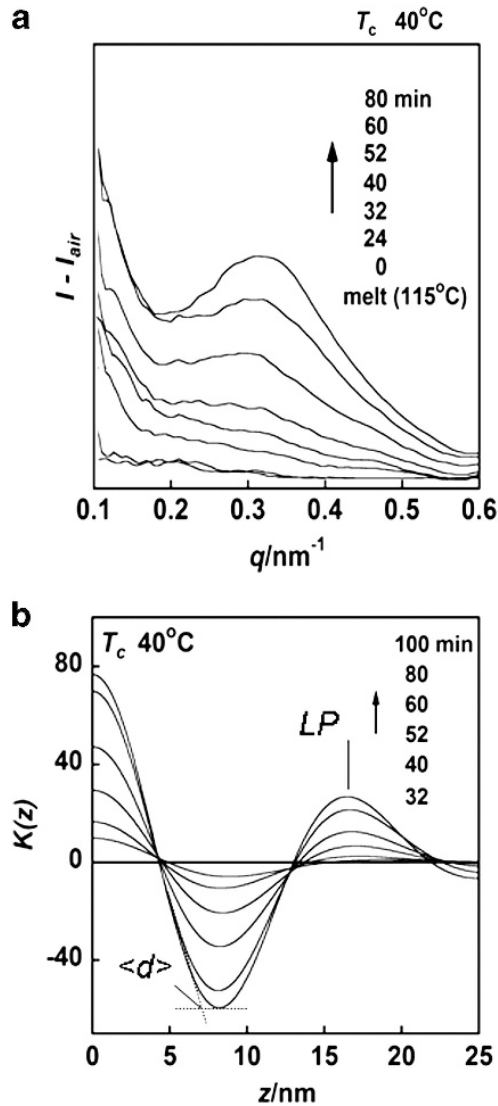

Figure 3 (a) Time dependence of the SAXS profiles of TPI measured in the isothermal crystallization process at $T_{C}=40^{\circ} \mathrm{C}$ from the melt. The airscattering component $\left(I_{\text {air }}\right)$ was subtracted. (b) Correlation functions of the stacked lamellar structure calculated from the SAXS data in the $q$ range of the LP peak.

which corresponded to the IR $2603 \mathrm{~cm}^{-1}$ band, first appeared from the melt and later transferred to the $\alpha$-crystalline phase.

In the crystalline $\alpha$-bands, some bands appeared at a different time from the others. For example, the detection of the $1205 \mathrm{~cm}^{-1}$ band started at approximately $20 \mathrm{~min}$, which was almost the same time as the decrement of the $2603 \mathrm{~cm}^{-1}$ band intensity. The $\alpha$-form band at $1050 \mathrm{~cm}^{-1}$ appeared at approximately $32 \mathrm{~min}$, precisely when the WAXD peak of the $\alpha$-crystal lattice started to be detected. The time gap between these two crystallization-sensitive bands may be reasonably interpreted using the concept of critical sequence length. ${ }^{39,40}$ The infrared bands of the helical chain conformation can be detected when the length of the regular helix exceeds a critical value that is intrinsic to the individual vibrational mode. The band corresponding to the shorter critical sequence length should appear earlier than the band with the longer critical sequence length, which reflects the growth of the regular helix. ${ }^{41}$ Thus, the band at $1205 \mathrm{~cm}^{-1}$ corresponds to the shorter helix, and the band at $1050 \mathrm{~cm}^{-1}$ corresponds to the longer one, although the concrete minimal number of monomeric units to make a detectable helical segment must be evaluated experimentally using the isotope dilution method. ${ }^{39}$ It must be noted here that the WAXD peak appeared at approximately the same time as the infrared $1050 \mathrm{~cm}^{-1}$ band with the long critical sequence length, which indicates that the crystalline lattice started forming when relatively long helical segments gather together side by side. 


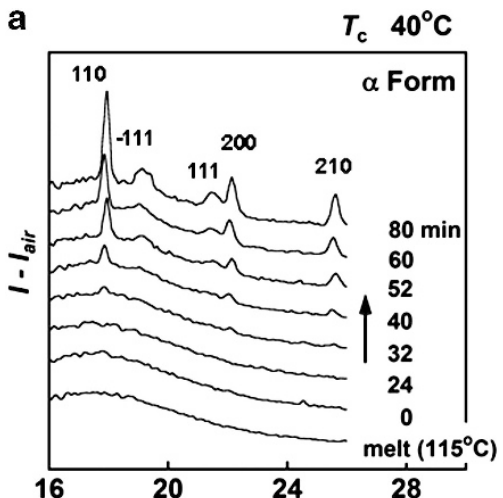

b
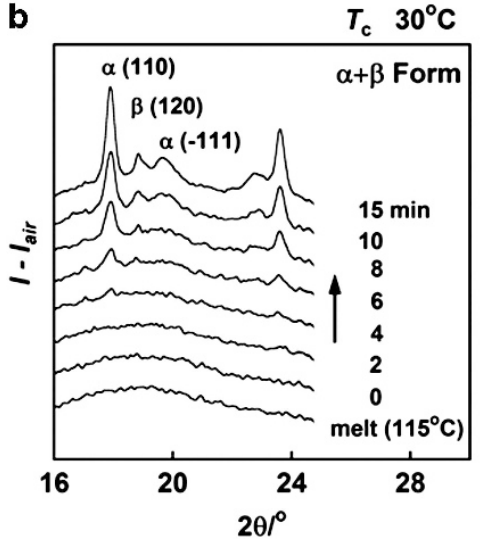

Figure 4 Time dependence of the WAXD profiles of TPI measured in the isothermal crystallization process at (a) $T_{c}=40^{\circ} \mathrm{C}$ and (b) $T_{C}=30^{\circ} \mathrm{C}$ from the melt. In $\mathbf{a}$, the indices are of the crystalline $\alpha$-form. ${ }^{35}$
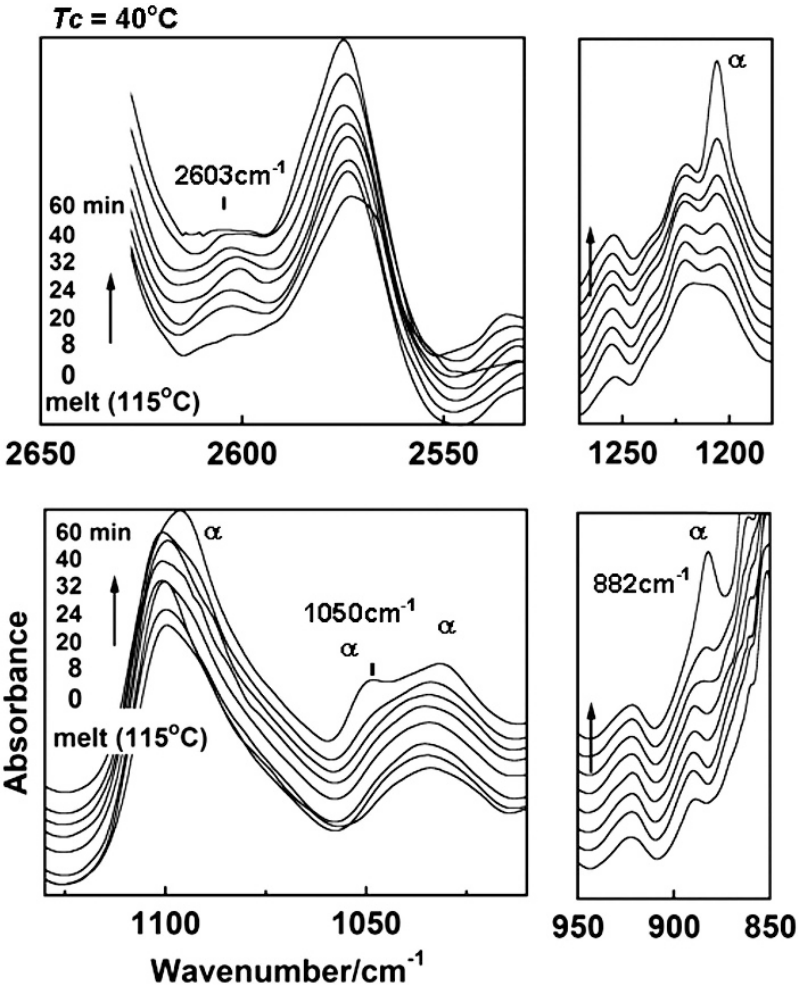

Figure 5 Time dependence of the FTIR spectra of TPI measured in the isothermal crystallization process at $T C=40^{\circ} \mathrm{C}$ from the melt.

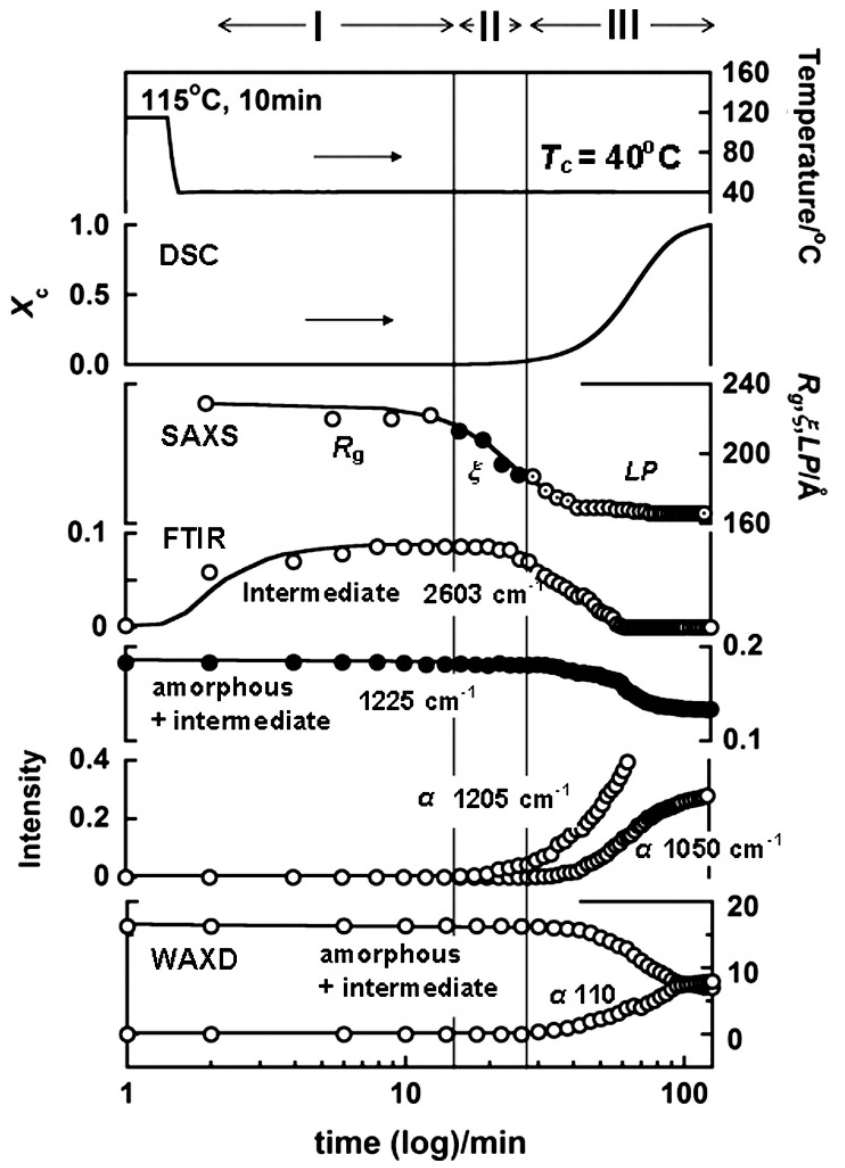

Figure 6 Time dependence of various experimental data that were obtained from the simultaneous FTIR/WAXD/SAXS measurements performed for TPI in the isothermal crystallization process at $T_{c}=40^{\circ} \mathrm{C}$ from the melt.

As observed in Figure 6, the amorphous region, which is represented by the infrared band at $1225 \mathrm{~cm}^{-1}$ and the WAXD halo component, did not decrease its population until the $\alpha$-crystalline peaks appeared, although most of the sample was already in the intermediate phase. In other words, the observed WAXD halo peak might be similar between the intermediate phase and the pure amorphous phase, although some of the infrared bands were different between them. Therefore, we speculated that the intermediate phase consisted of a random aggregation of short helical segments, which resulted in a slightly higher density than the molten state, as we observed from the SAXS component discussed below.

Figure 3a shows the time dependence of the SAXS profiles. In the data analysis of the observed SAXS data, we assumed that the crystallization process might be divided into three time regions: (i) the time region immediately after the temperature jump, (ii) the time region before the appearance of lamellae and (iii) the time region of the lamellar structure formation, as seen in several examples of polymers such as nylon and isotactic polypropylene. ${ }^{41,42}$ As reported in previous works, region (i) may be analyzed using a Guinier plot $\left[\ln (I)\right.$ vs $\left.q^{2}\right]$, but the $q$ range observed in the experiment did not always satisfy the effective condition for this approximated equation $\left(q<<R_{g}{ }^{-1}\right.$, where $R_{g}$ is a radius of gyration). Hence, the equation of power function of $q$ was used as shown below. ${ }^{43}$ The scattering intensity from an isolated particle with the correlation 


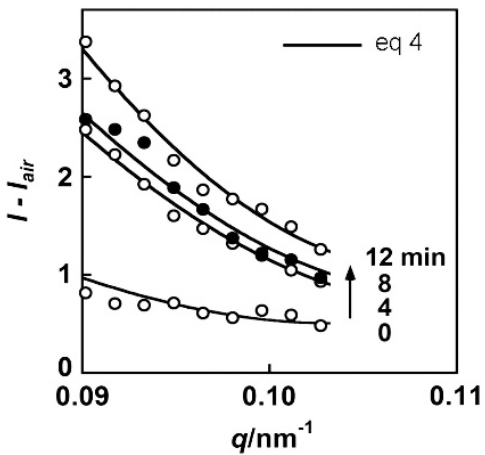

Figure 7 SAXS intensity $/(q)$ plotted against the scattering vector $q$ at various times in time region I, which was evaluated using the SAXS data of TPI taken in the isothermal crystallization process at $T_{c}=40^{\circ} \mathrm{C}$ from the melt. The solid lines are the curves calculated using equation 4 in the text.

function $\gamma(r)$ is given as:

$$
I(q)=4 \mathrm{p} \int_{0}^{\infty} \gamma(r)[\sin (q r) /(q r)] r^{2} \mathrm{~d} r
$$

Because the following approximation may be possible,

$$
\sin (q r) /(q r) \approx 1-(q r)^{2} / 6+(q r)^{4} / 120
$$

equation 2 was approximated by the following form, which consists of the second- and forth-order terms and can be applied to a wider $q$ range than the Guinier plot.

$$
I(q) \approx I(0)\left[1-\left(M_{4} / 6 M_{2}\right) D^{2} q^{2}+\left(M_{6} / 120 * M_{2}\right) D^{4} q^{4}\right]
$$

where $D$ is the longest length of the particle and is equal to the radius of a spherical model. $M_{\mathrm{k}}$ is the $k$-th moment of the correlation function $\gamma(r)$.

$$
M_{\mathrm{k}}=D^{-(k+1)} \int_{0}^{\mathrm{D}} r^{k} g(r) \mathrm{d} r
$$

The radius of gyration $R_{g}$ can be calculated as below.

$$
R_{\mathrm{g}}=\left(M_{4} / 2 M_{2}\right)^{1 / 2} \mathrm{D}
$$

The SAXS intensity data in the $q$ range $<0.12 \mathrm{~nm}^{-1}$ were analyzed using equation (4). The observed intensity $I(q)$ was plotted against $q$ (Figure 7), and the adjustable coefficients of equation (4) were determined by fitting the observed data points to this equation. The observation of $R_{g}$ corresponds to the formation of domains with higher densities than the surrounding molten region. In the time region (ii), these domains were assumed to approach each other with a correlation length $\xi$ to form the aggregation state. The so-called Debye-Bueche equation was applied here to estimate the correlation length $\xi .44,45$ For the correlation function of $g(r)=\exp (-r / \xi)$, we have

$$
I(q)=A /\left(1+\xi^{2} q^{2}\right)^{2}
$$

where $A$ is a constant. Although this equation may, in principle, be applicable to any $q$ range, the data analysis must avoid the disturbance by the low-angle scatterings from the newly generated domains and the wider-angle scatterings due to the inner structure of each domain. In the actual analysis of equation (6), $I(q)^{-1 / 2}$ was plotted against $q^{2}$ in the $q$ range of $0.15-0.17 \mathrm{~nm}^{-1}$ and the slope of the straight line provided the $\xi^{2}$ value (Figure 8 ). The obtained $\xi$ values are plotted in Figure 6.

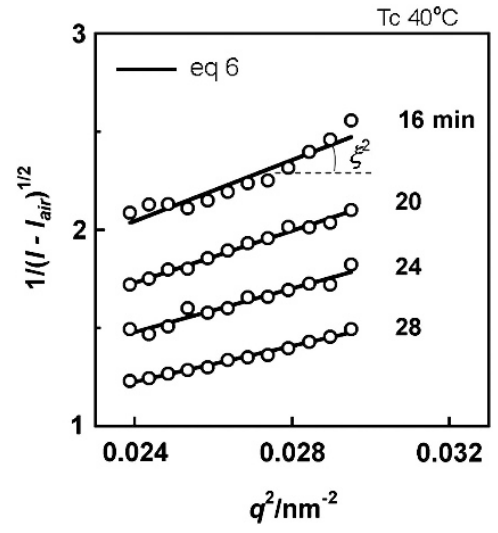

Figure 8 Debye-Bueche plots made for the SAXS intensity $I(q)$ at various times in time region II of the isothermal crystallization process of TPI at $T_{C}=40^{\circ} \mathrm{C}$ from the melt.

In region (iii), these domains were assumed to join together to form the lamellar structure with the long period (LP). The correlation function corresponding to the stacked lamellar structure was applied using the following equation. ${ }^{42}$

$$
K(z)=\left\langle\left[\eta\left(z^{\prime}\right)-\langle\eta\rangle\right]\left[\eta\left(z+z^{\prime}\right)-\langle\eta\rangle\right]\right\rangle=2(\pi)^{-1} \int_{0}^{\infty} q^{2} I(q) \cos (q z) d q
$$

where $\eta(z)$ is the electron density at position $z$ along the direction normal to the stacked lamellar planes, and $\langle\eta\rangle$ is the ensemble average. Some examples of the correlation curves are shown in Figure $3 \mathrm{~b}$. The LP and averaged lamellar thickness $\langle d\rangle$ were obtained from the points shown on these curves.

Figure 6 shows the structural parameters obtained from the abovementioned three types of SAXS equations in comparison with the FTIR and WAXD data at $T_{c}=40{ }^{\circ} \mathrm{C}$. After the temperature jump, the domains with $R_{g}$ of approximately $220 \AA$ were generated, and the infrared band at $2603 \mathrm{~cm}^{-1}$ started to increase in intensity. As discussed before, this band was assigned to a disordered intermediate phase, which is the intermediate phase where the not-regular but notperfectly-disordered domains of approximately $220 \AA$ radii appeared in the melt and had a higher density than the surrounding melt. At the starting point of time region II, the correlation length $\xi$ of the neighboring domains was approximately the same as $R_{g}$, and then $\xi$ decreased gradually and reached the LP of the stacked lamellar structure when time region III started. During these processes, the FTIR data showed the generation of the conformationally disordered helical chain segments in the melt and the growth of these chains in the intermediate-phase domains (region I). As observed in the $1205 \mathrm{~cm}^{-1}$ band, these regular chain segments continued growing in region II and finally formed the crystalline lattice of the $\alpha$-form in region III.

Figure 6 shows that $R_{\mathrm{g}}, \xi$ and LP changed continuously with time, which suggests the continuous transformation of a higher-order structure. Figure 9 illustrates an explanation for these relations. After the domains of $R_{\mathrm{g}}$ size appeared, the centers of gravity of the neighboring domains approached one another, and the strong correlation started when the distance between the domain centers was approximately equal to $R_{\mathrm{g}}(=\xi)$. Various correlation distances $\left(\xi_{1}, \xi_{2}, \ldots\right)$ were detected among these domains. The observable shortest correlation length $\xi_{\min }$ in the present SAXS experiment may be dependent on the resolution of the SAXS 

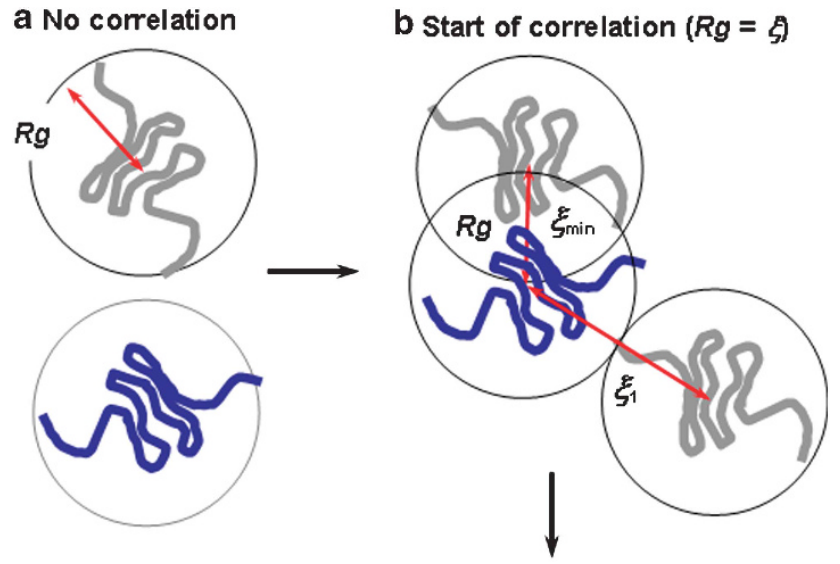

d Formation of
stacked lamellae
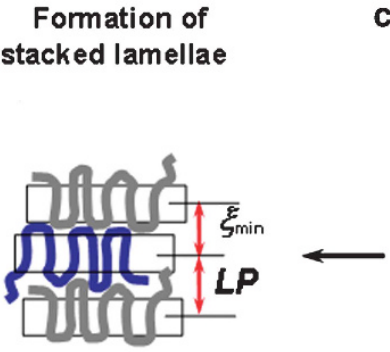

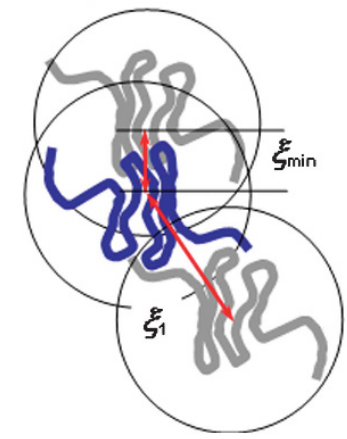

Figure 9 Illustration of the relationship between $R_{\mathrm{g}}$, $\xi$ and LP estimated from the SAXS data.

system or on the limitation of the detectable $q$ range. In other words, the longer correlation lengths cannot be detected in this SAXS experiment because of the limited $q$ range. Thus, we have a relation such that

$$
q_{\mathrm{obs}} \approx 1 /<\xi>\approx 1 / \xi_{1}+1 / \xi_{2}+\ldots \approx 1 / \xi_{\min }
$$

where $\langle\xi\rangle$ is the average of all $\xi_{\mathrm{i}}$ values $(\mathrm{i}=1,2, \ldots)$. These domains aggregated together, and the detectable correlation length $\left(\xi_{\min }\right)$ decreased with time; finally, the domains were connected into the stacked lamellar structure with the LP.

A concrete image of the structural evolution process in the isothermal crystallization at $T_{c}=40{ }^{\circ} \mathrm{C}$ is illustrated in Figure 10 . In the molten state, the molecular chains might be disordered. They were approximately regularized to form the intermediate phase, which consisted of domains of approximately $220 \AA$ radius. As shown in Figures 1 and 2, the infrared band at $2603 \mathrm{~cm}^{-1}$ appeared from the melt and increased in intensity. Afterwards, the intensity of this band decreased and the bands of the $\alpha$-crystalline form appeared. The $2603 \mathrm{~cm}^{-1}$ band was considered to correspond to the third phase that was different from both the melt and the $\alpha$ crystalline form, and this third phase is named the intermediate phase, as previously explained. The time region of the observation of the intermediate phase corresponds to the appearance of the domains in the SAXS data. Previously, the infrared bands that are intrinsic to the $\alpha$-form had a regular helical chain conformation, which suggests that the molecular chain segments in the intermediate phase or in the domains might be disordered in conformation, but they gathered together to form a relatively higher

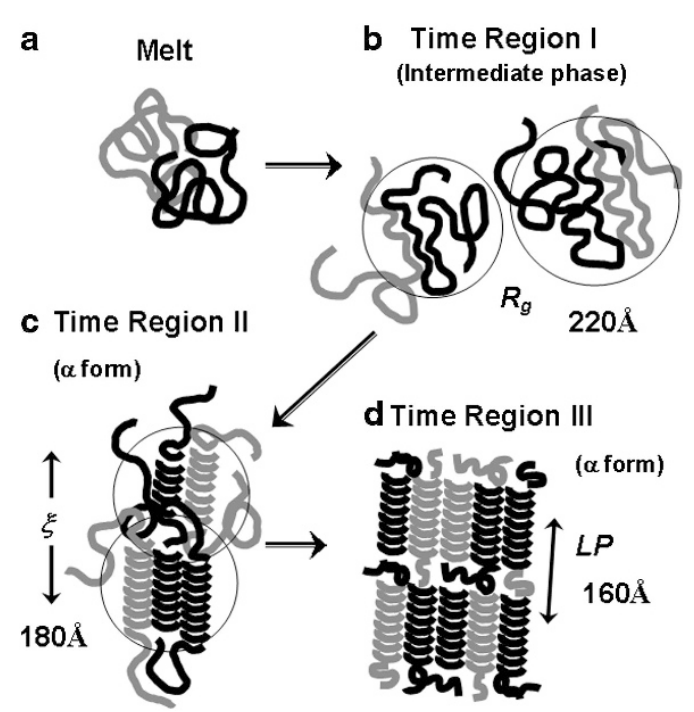

Figure 10 Schematically illustrated structural evolution model derived for the isothermal crystallization process of TPI at $T_{c}=40^{\circ} \mathrm{C}$ from the melt: (a) the random coils in the melt, (b) the generation of domains with $R_{g}$ of approximately $220 \AA$, (c) the correlation of domains with the correlation length $\xi$ and (d) the formation of stacked lamellar structures with the LP.

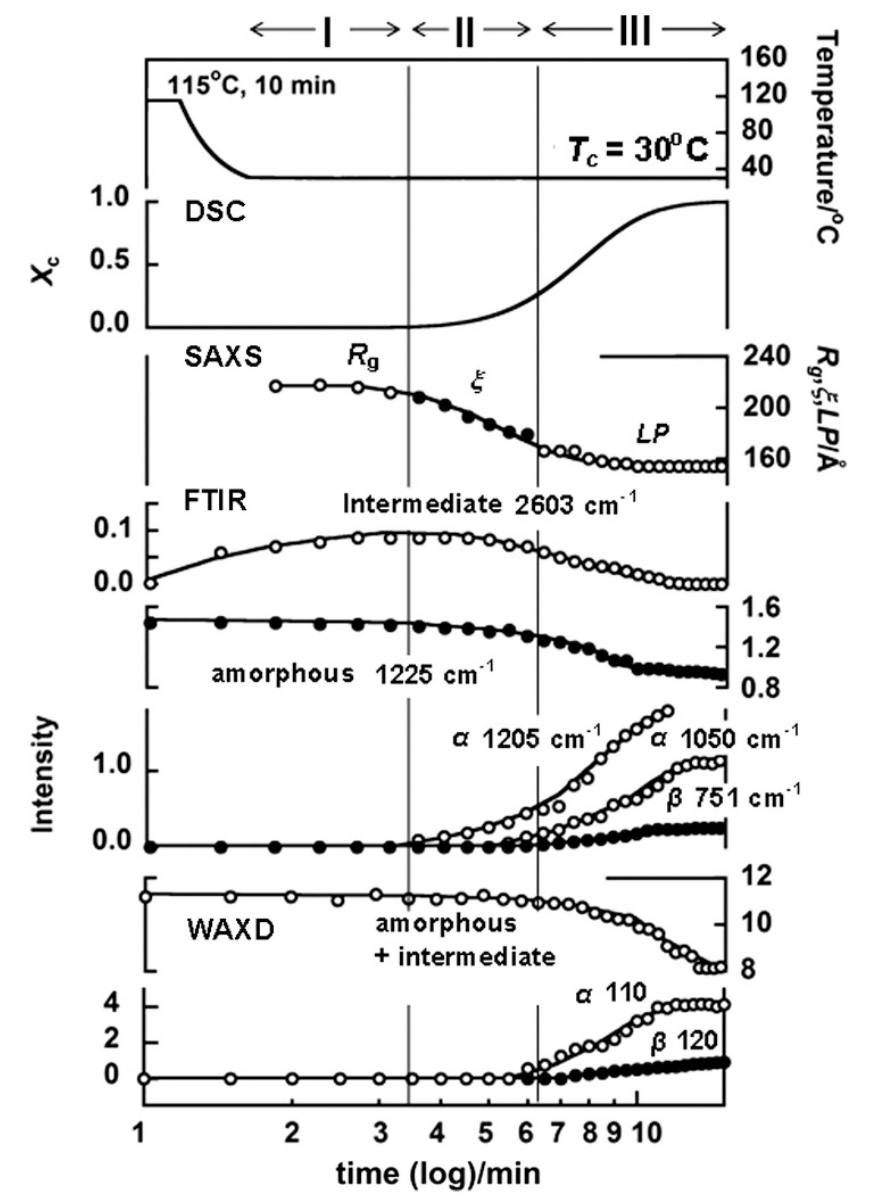

Figure 11 Time dependence of various experimental data obtained from the simultaneous FTIR/WAXD/SAXS measurements performed for TPI in the isothermal crystallization process at $T_{C}=30^{\circ} \mathrm{C}$ from the melt. 


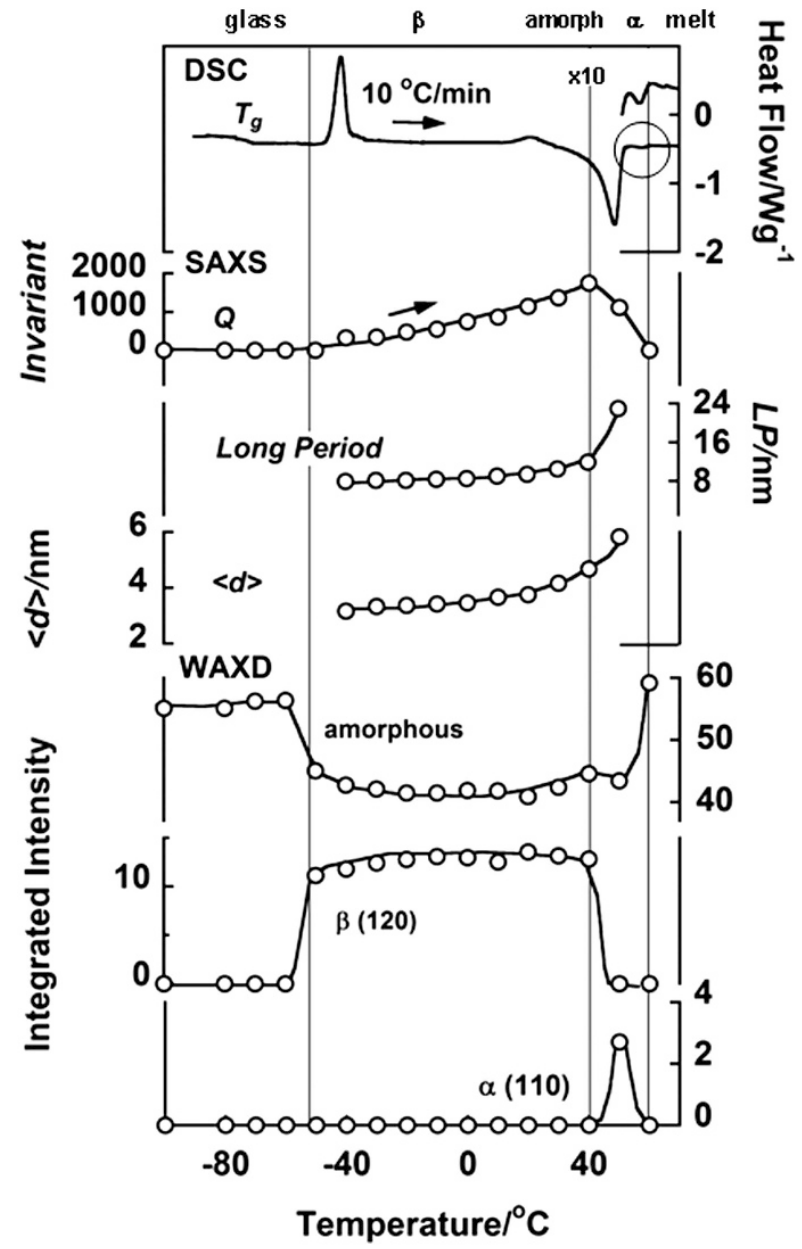

Figure 12 Temperature dependence of various parameters estimated from the SAXS, WAXD and FTIR data collected in the cold-crystallization process of TPI from the amorphous state at $-100^{\circ} \mathrm{C}$.

density region. As time passed, these domains approached each other to form the stacked lamellar structure at the final stage, where the conformationally regular chains of the $\alpha$-form gathered in parallel to form the crystalline lattice.

(B) $T_{c}=30^{\circ} \mathrm{C}$

A similar structural evolution was observed in the case of isothermal crystallization at $T_{c}=30{ }^{\circ} \mathrm{C}$ but in a shorter time scale, as shown in Figure 11. In contrast to the case of $T_{c}=40^{\circ} \mathrm{C}$, the crystalline $\beta$-form was determined to crystallize in addition to the $\alpha$ form, as observed from both the infrared data and the WAXD data (Figure $4 \mathrm{~b}$ ). The $\beta$-form appeared a short time after the $\alpha$-form. As a trial, the Avrami-type plot was made using the integrated intensity of various crystallization-sensitive infrared bands of the $\alpha$ - and $\beta$-forms. The averaged $n$ value estimated for the $\alpha$-bands at $T_{c}=40^{\circ} \mathrm{C}$ was 2.49 , which is approximately the same as the value 2.45 obtained by the DSC data analysis. The rate constant $\log k=-3.54$, which was estimated for the $\alpha$-bands, is also similar to the value -3.57 derived from the DSC data analysis at $T_{c}=40{ }^{\circ} \mathrm{C}$. At $T_{c}=30^{\circ} \mathrm{C}$, the averaged $n$ value was 2.18 for the $\alpha$-form and 2.80 for the $\beta$-form. The $\alpha$-form appeared approximately $4 \mathrm{~min}$ after the temperature jump, and the $\beta$-form appeared at approximately $5.5 \mathrm{~min}$. Thus, the induction time was different between these two crystalline phases, which indicates the difference in the nucleation rate at the early stage of crystallization. When the crystallization temperature was lower, the fraction of the $\beta$-form was increased with a shorter induction time.

\section{Cold crystallization}

The molten sample was quenched at liquid nitrogen temperature to obtain the glass sample. Then, the sample was set to a metal holder by keeping the temperature below $-100{ }^{\circ} \mathrm{C}$. The WAXD and SAXS patterns were measured in the stepwise heating process (approximately $2^{\circ} \mathrm{C} \mathrm{min}^{-1}$ in average). Figure 12 summarizes these experimental data in comparison with the DSC thermogram (collected at $\left.10^{\circ} \mathrm{C} \mathrm{min}^{-1}\right)$. In the WAXD data, the initial amorphous halo pattern changed to that of the $\beta$-form at approximately $-50{ }^{\circ} \mathrm{C}$, where an exothermic peak was detected in the DSC thermogram. The crystallized $\beta$-form was stable up to $50{ }^{\circ} \mathrm{C}$, where it sharply disappeared, and the amorphous phase appeared in a narrow temperature region once and then changed to the $\alpha$-form. The $\alpha$-form was melted at a higher temperature that corresponded to a small endothermic peak of the DSC thermogram. (Strictly speaking, a small temperature deviation might be detected between the DSC peak and the melting of the $\alpha$-form because of the difference in heating rate). The analysis of the SAXS data revealed the temperature dependence of the invariant $Q$, the LP and the lamellar thickness $<d>$. With an increase of temperature, the lamellar thickness and the LP increased gradually; then, they increased remarkably in the $\alpha$ form formation region.

\section{CONCLUSIONS}

In this paper, simultaneous measurements were performed for WAXD and SAXS and for WAXD, SAXS and FTIR data, from which the microscopically viewed structural changes were clarified regarding the crystallization processes of TPI. In the melt-isothermal crystallization process at $40^{\circ} \mathrm{C}$, an intermediate phase appeared from the melt and later transformed to the $\alpha$-form. At $T_{c}=30^{\circ} \mathrm{C}$, the situation was similar, but the crystallization to the $\beta$-form was included in addition to the $\alpha$-form. The combination of the SAXS, WAXD and FTIR data allowed us to derive the structural evolution process from various levels as illustrated in Figure 10. In the coldcrystallization process from the glass sample, the crystallization into the $\beta$-form occurred. The $\beta$-form transformed to the regular $\alpha$-form via the intermediate phase during the heating process to the molten state.

Therefore, the crystallization behavior that started from the melt is different from the behavior that started from the glass. The $\alpha$-form seems stable at high temperatures, whereas the $\beta$-form is unstable at high temperatures and melts at a relatively lower temperature of approximately $50{ }^{\circ} \mathrm{C}$. The once-melted sample transforms again to the crystalline $\alpha$-form at a higher temperature.

In this paper, we did not clarify the characteristic structural features of the $\alpha$ - and $\beta$-forms that we detected in various circumstances: these crystalline forms may change the regularities in chain-conformation and chain-packing mode depending on the preparation conditions. Using the oriented samples, we can obtain more detailed information about these irregular-regular transitions in the heating and cooling processes, as we will report in a separate paper.

\section{ACKNOWLEDGEMENTS}

This study was supported financially by MEXT 'Strategic Project to Support the Formation of Research Bases at Private Universities (2010-2014)'. 
1 Tashiro, K., Yamamoto, H., Yoshioka, T., Ninh, T. H., Shimada, S., Nakatani, T., Iwamoto, H., Ohta, N. \& Masunaga, H. Development of simultaneous measurement system of wide- and small-angle X-ray scattering and vibrational spectra for the static and dynamic analyses of hierarchical structures of polymers. KOBUNSHIRONBUNSHU (special issue) 69, 213-227 (2012).

2 Tashiro, K. \& Sasaki, S. Structural changes in the ordering process of polymers as studied by an organized combination of the various measurement techniques. Prog. Polym. Sci. 28, 451-519 (2003).

3 Tashiro, K., Nakamura, A., Yoshioka, T., Yamamoto, H., Ninh, T. H., Masunaga, H. \& Sasaki, S. Development of simultaneous measurement system of transmission FTIR, wide-angle and small-angle X-ray scatterings (3) construction of computer-controlled temperature-jump cell and its application to the study of isothermal crystallization phenomenon of polyethylene melt. Fiber Preprints, Japan 67, 192 (2012).

4 Blum, J., Parahy, E. \& Machtou, P. Warm vertical compaction sequences in relatin to gutta-percha temperature. J. Endod. 23, 307-311 (1997).

5 Song, J., Huang, B. \& Yu, D. Progress of synthesis and application of trans-1,4polyisoprene. J. Appl. Polym. Sci. 82, 81-89 (2001).

6 Tsukada, G., Tanaka, T., Torii, M. \& Inoue, K. Shear modulus and thermal properties of gutta percha for root canal filling. J. Oral Rehabil. 31, 1139-1144 (2004).

7 Miner, M. R., Berzins, D. W. \& Bahcall, J. K. A comparison of thermal properties between gutta-percha and a synthetic polymer based root canal filling material (resilon). J. Endod. 32, 683-686 (2006).

8 Silva, J. B. A., de Paula, R. C. M., Feitosa, J. P. A., Gurgel-Filho, E. D., Ferreira, C. M. \& Filho, F. J. S. In Vivo aging of gutta-percha dental cone. J. Appl. Polym. Sci. 100, 4082-4088 (2006).

9 Zhao, Y., Huang, B., Yao, W., Cong, H., Shao, H. \& Du, A. Epoxidation of high trans1,4-polyisoprene and its properties. J. Appl. Polym. Sci. 107, 2986-2993 (2008).

10 Tully, J. A Victorian ecological disaster: imperialism, the Telegraph, and gutta percha. J. World Hist. 20, 559-579 (2009).

11 Zhang, J. \& Xue, Z. A comparative study on the properties of Eucommia ulmoides gum and synthetic trans-1,4-polyisoprene. Poly. Test. 30, 753-759 (2011).

12 Tangpakdee, J., Tanaka, Y., Shiba, K., Kawahara, S., Sakurai, K. \& Suzuki, Y. Structure and biosynthesis of trans-polyisoprene from Eucommia ulmoides. Phytochemistry 45 75-80 (1997).

13 Bamba, T., Fukusaki, E., Nakazawa, Y. \& Kobayashi, A. In-situ chemical analyses of trans-polyisoprene by histochemical staining and Fourier tansform infrared microspectroscopy in a rubber-producing plant, Eucommia ulmoides oliver. Planta 215, 934-939 (2002)

14 Warneke, S., Arenskötter, M., Tenberge, K. B. \& Steinbüchel, A. Bacterial degradation of poly(trans-1,4-isoprene) (gutta percha). Microbiology 153, 347-356 (2007).

15 Takeno, S., Bamba, T., Nakazawa, Y., Fukusaki, E., Okazawa, A. \& Kobayashi, A. A high-throughput and solvent-free method for measurement of natural polyisoprene content in leaves by Fourier transform near infrared spectroscopy. J. Biosci. Bioeng. 106, 537-540 (2008).

16 Zinck, P., Terrier, M., Mortreux, A. \& Visseaux, M. On the number-average molecular weight of poly(1,4-trans isoprene) determined by conventional GPC. Polym. Test. 28, 106-108 (2009).

17 Bunn, C. W. Molecular structure and rubber-like elasticity. I. The crystal structures of $\beta$ gutta percha, rubber and polychloroprene. Proc. R. Soc. Lond. A 180, 40-66 (1942). 18 Fisher, D. Crystal structure of gutta percha. Phys. Proc. Soc. 66, 7-12 (1952).

19 Takahashi, Y., Sato, T. \& Tadokoro, H. Crystal structure of $\alpha$-gutta percha: modification of the constrained least-squares method. J. Polym. Sci. Polym. Phys. Ed. 11, 233-248 (1973).

20 Su, F., Yan, D., Liu, L., Luo, J., Zhou, E. \& Qian, R. A new crystal modification of gutta percha. Polymer (Guildf) 39, 5379-5385 (1998).

21 Fischer, E. \& Henderson, J. F. Study of crystallization kinetics of trans-1,4-polyisoprene. J. Polym. Sci. Part A2 5, 377-390 (1967).

22 Lovering, E. G. \& Wooden, D. C. Transition in trans-1,4-polyisoprene. J. Polym. Sci. Part A2 7, 1639-1649 (1969).
23 Schilder, H., Goodman, A. \& Aldrich, W. The thermomechanical properties of guttapercha III. Determination of phase transition temperatures for gutta-percha. Oral Surg. 38, 109-114 (1974).

24 Rootare, H. M. \& Powers, J. M. Determination of phase transitions in gutta-percha by differential thermal analysis. J. Dent. Res. 56, 1453-1462 (1977).

25 Martuscelli, E. Annealing behaviour of melt crystallized trans-1,4-polyisoprene. Die Makromolekulare Chemie 151, 159-168 (1972).

26 Hardin, I. R. \& Yeh, G. S. Y. Strain-induced crystallization and reversible phenomena in trans-polyisoprone II. X-ray studies. J. Macromol. Sci. Part B. 7, 393-409 (1973)

27 Yeh, G. S. Y. Thermal reversibility of crystal surface structure and its contribution to degree of crysallinity in natural rubber and trans-polyisoprene. J. Macromol. Sci. Part B 8, 241-257 (1973).

28 Anandakumaran, K., Kuo, C. C., Mukherji, S. \& Woodward, A. E. Crystallization of trans-1,4-polyisoprene. J. Polym. Sci.: Polym. Phys. Ed. 20, 1669-1676 (1982).

29 Cooper, W. \& Vaughan, G. Crystallization of gutta percha and synthetic trans-1,4polyisoprenes. Eleventh Canadian High Polymer Forum 329-340 (1962).

30 Lovering, E. G. The relationship between molecular weight and spherulitic growth rates in trans-1,4-polyisoprene. J. Poly. Sci. Part C 30, 329-338 (1970).

31 Burfield, D. R. \& Lim, K. L. Differential scanning calorimetry analysis of natural rubber and related polyisoprenes. measurement of the glass transition temperature. Macromolecules 16, 1170-1175 (1983).

32 Arvanitoyannis, I. \& Blanshard, J. M. V. Crystallization kinetics of native (pure) and commercial gutta percha (trans-polyisoprene) and the influence of metal salts, oxides, 'thermoplast' and colouring agents on its crystallization rate. Poly. Int. 27, 297-303 (1992).

33 Vasanthan, N., Corrigan, J. P. \& Woodward, A. E. Infra-red spectroscopic investigation of bulk-crystallized trans-1,4-polyisoprene. Polymer (Guildf) 34, 2270 2276 (1993).

34 Cerveny, S., Zinck, P., Terrier, M., Arrese-Igor, S., Alegria, A. \& Colmenero, J. Dynamics of amorphous and semicrystalline 1,4-trans-poly(isoprene) by dielectric spectroscopy. Macromolecules 41, 8669-8676 (2008).

35 Ratri, P. J., Tashiro, K. \& Iguchi, M. Experimentally- and theoretically-evaluated ultimate 3-dimensional elastic constants of trans-1,4-polyisoprene $\alpha$ and $\beta$ crystalline forms on the basis of the newly-refined crystal structure information. Polymer (Guildf) 53, 3548-3558 (2012).

36 Avrami, M. Kinetics of phase change. I General theory. J. Chem. Phys. 7, 1103 (1939).

37 Avrami, M. Kinetics of phase change II. Transformation-time relations for random distribution of nuclei. J. Chem. Phys. 8, 212 (1940).

38 Avrami, M. Granulation, phase change, and microstructure kinetics of phase change III. J. Chem. Phys. 9, 177 (1941).

39 Kobayashi, M., Akita, K. \& Tadokoro, H. Infared spectra and regular sequence lengths in isotactic polymer chains. Die Makromol. Chem. 118, 324-362 (1968).

40 Kobayashi, M. Roles of polymer-solvent complexes in conformational ordering of polymer molecules dispersed in gels. Macromol. Symposia 114, 1-12 (1997).

41 Reddy, R. K., Tashiro, K., Sakurai, T., Yamaguchi, N., Sasaki, S., Masunaga, H. \& Takata, M. Isothermal crystallization behavior of isotactic polypropylene H/D blends as viewed from time-resolved FTIR and synchrotron SAXS/WAXD measurements. Macromolecules 42, 4191-4199 (2009).

42 Tashiro, K., Reddy, K. R., Nishiyama, A., Hanesaka, M., Tsuji, S., Hashida, T., Weiyu C., Masunaga, H., Sasaki, S. \& Takata, M. Evolution process of regular structure in isothermal crystalline polymers viewed from synchrotron small- and wide-angle x-ray scatterings and vibrational spectroscopy. Kobunshi Ronbunshu 66, 536-549 (2009).

43 Feigen, L. A. \& Svergun, D. I. Structure Analysis by Small-Angle X-ray and Neutron Scattering 59-76 (Plenum Press, New York, 1987).

44 Debye, P. \& Bueche, A. M. Scattering by an inhomogeneous solid. J. Appl. Phys. 20, 518 (1949).

45 Glatter, O. \& Kratky, O. Small Angle X-ray Scattering 446-449 (Academic Press, London, 1982). 